\title{
Some Improvements of Hölder's Inequality on Time Scales
}

\author{
Cristian Dinu, Mihai Stancu and Daniela Dănciulescu
}

\begin{abstract}
The theory and applications of dynamic derivatives on time scales have recently received considerable interest. In this paper, we define a function using the combined diamond- $\alpha$ integral, investigate its monotonicity and give some refinements for Hölder's inequality. We present some applications for $\mathbb{Z}$ and $\mathbb{R}$.
\end{abstract}

\section{Introduction}

There have been recent developments of the theory and applications of dynamic derivatives on time scales. This study, which is an unification of the discrete theory with the continuous theory, provides an unification and extension of traditional differential and difference equations. It is also an important tool in many computational and numerical applications. Using the $\Delta$ (delta) and $\nabla$ (nabla) dynamic derivatives, a combined dynamic derivative, so called $\nabla_{\alpha}$ (diamond- $\alpha$ ) dynamic derivative, was introduced as a linear combination of $\Delta$ and $\nabla$ dynamic derivatives on time scales. The diamond- $\alpha$ derivative reduces to the $\Delta$ derivative for $\alpha=1$ and to the $\nabla$ derivative for $\alpha=0$. We refer the reader to [2], [4] and [5] for an account of the calculus associated with the diamond- $\alpha$ dynamic derivatives.

Recently, it has been proven a complete weighted version of diamond- $\alpha$ of Jensen's Inequality (see [3]) :

Key Words: Time scales, convex function, dynamic derivatives, Hölder's inequality. 2010 Mathematics Subject Classification: Primary 26D15; Secondary 39A13.

Received: 25.11 .2016

Revised: 15.11 .2016

Accepted: 21.12.2016 
Theorem 1. [3, Theorem 2]. Let $a, b \in \mathbb{T}$ and $m, M \in \mathbb{R}$. If $g \in C\left([a, b]_{\mathbb{T}},[m, M]\right)$ and $w \in C\left([a, b]_{\mathbb{T}}, \mathbb{R}\right)$ with $\int_{a}^{b} w(t) \diamond_{\alpha} t>0$, then the following assertions are equivalent:

(i) $w$ is an $\alpha-S P$ weight for $g$ on $[a, b]_{\mathbb{T}}$;

(ii) for every $F \in C([m, M], \mathbb{R})$ convex function, we have

$$
F\left(\frac{\int_{a}^{b} g(t) w(t) \diamond_{\alpha} t}{\int_{a}^{b} w(t) \diamond_{\alpha} t}\right) \leq \frac{\int_{a}^{b} F(g(t)) w(t) \diamond_{\alpha} t}{\int_{a}^{b} w(t) \diamond_{\alpha} t} .
$$

This version of Jensen's inequality is complete since it is true if and only if $w$ is an $\alpha-S P$ weight for $g$ and this special class of weights allows them to take some negative values.

Using Theorem 1, we can obtain stronger versions of many important results, such as Hölder's inequality.

Theorem 2. (Hölder's inequality). Let $\mathbb{T}$ be a time scale, $a<b \in \mathbb{T}, f, g \in$ $C\left([a, b]_{\mathbb{T}},[0,+\infty)\right)$ and $w \in C\left([a, b]_{\mathbb{T}}, \mathbb{R}\right)$ such that $\mathrm{wg}^{q}$ is an $\alpha-S P$ weight for $f g^{-\frac{q}{p}}$, where $p$ and $q$ are Hölder conjugates, (that is, $\frac{1}{p}+\frac{1}{q}=1$ ) and $p>1$. Then, we have

$$
\int_{a}^{b} w(t) f(t) g(t) \diamond_{\alpha} t \leq\left(\int_{a}^{b} w(t) f^{p}(t) \diamond_{\alpha} t\right)^{\frac{1}{p}}\left(\int_{a}^{b} w(t) g^{q}(t) \diamond_{\alpha} t\right)^{\frac{1}{q}} .
$$

If $p<1$, then the inequality (2) is backward.

Proof. We choose $F(x)=x^{p}$ in Theorem 2, and $F$ is a convex function on $[0, \infty)$ for $p>1$. We get

$$
\left(\frac{\int_{a}^{b} g(t) w(t) \diamond_{\alpha} t}{\int_{a}^{b} w(t) \diamond_{\alpha} t}\right)^{p} \leq \frac{\int_{a}^{b}(g(t))^{p} w(t) \diamond_{\alpha} t}{\int_{a}^{b} w(t) \diamond_{\alpha} t}
$$

Since $w g^{q}$ is an $\alpha-S P$ weight for $f g^{-\frac{q}{p}}$, we have

$$
\left(\frac{\int_{a}^{b} w(t) g^{q}(t) f(t) g^{-q / p}(t) \diamond_{\alpha} t}{\int_{a}^{b} w(t) g^{q}(t) \diamond_{\alpha} t}\right)^{p} \leq \frac{\int_{a}^{b} w(t) g^{q}(t)\left(f(t) g^{-q / p}(t)\right)^{p} \diamond_{\alpha} t}{\int_{a}^{b} w(t) g^{q}\left(t \diamond_{\alpha} t\right.}
$$

But $p$ and $q$ are Hölder conjugates, and so 


$$
\int_{a}^{b} w(t) f(t) g(t) \diamond_{\alpha} t \leq\left(\int_{a}^{b} w(t) f^{p}(t) \diamond_{\alpha} t\right)^{\frac{1}{p}}\left(\int_{a}^{b} w(t) g^{q}(t) \diamond_{\alpha} t\right)^{\frac{1}{q}} .
$$

In section 2, we give our main results, regarding the function defined using Hölder's inequality. Some applications are presented in section 3.

\section{Main results}

Now, we will define a function based on Hölder's inequality, for time scales.

Thus, let $f, g$ and $w$ as in Theorem 2. We consider the function $h: \mathbb{T} \times \mathbb{T}$, given by

$$
\begin{aligned}
h(x, y)= & \left(\int_{x}^{y} w(t) f^{p}(t) \diamond_{\alpha} t\right)^{1 / p}\left(\int_{x}^{y} w(t) g^{q}(t) \diamond_{\alpha} t\right)^{1 / q} \\
& -\int_{x}^{y} w(t) f(t) g(t) \diamond_{\alpha} t .
\end{aligned}
$$

This difference is generated by the inequality (2), that gives the nonnegativity of the function $h$. We will study the monotonicity properties of $h$, and we will find some refinements of the inequality (2) based on his properties. These results generalize the ones obtained in [12].

The monotonicity properties of $h$ are given by the next theorem.

Theorem 3. Let $\mathbb{T}$ be a time scale, $a<b \in \mathbb{T}, f, g, w \in C\left([a, b]_{\mathbb{T}},[0,+\infty)\right)$, and $w \in C\left([a, b]_{\mathbb{T}}, \mathbb{R}\right)$ such that $w g^{q}$ is an $\alpha-S P$ weight for $f g^{-\frac{q}{p}}$, where $p$ and $q$ are Hölder conjugates. Then,

(i) if $p>1$, then the function $h(x, b)$ is monotonously decreasing, while if $p<1$, then the function $h(x, b)$ is monotonously increasing on $\mathbb{T}$, in relation with $x$;

(ii) if $p>1$, then the function $h(a, y)$ is monotonously increasing, while if $p<1$, then the function $h(a, y)$ is monotonously decreasing on $\mathbb{T}$ in relation with $y$;

(iii) for all $x \in \mathbb{T}, a<x<b$, if $p>1$, then: 


$$
\begin{aligned}
\int_{a}^{b} w(t) f(t) g(t) \diamond_{\alpha} t \leq & \left(\int_{a}^{x} w(t) f^{p}(t) \diamond_{\alpha} t\right)^{1 / p}\left(\int_{a}^{x} w(t) g^{q}(t) \diamond_{\alpha} t\right)^{1 / q} \\
& +\int_{x}^{b} w(t) f(t) g(t) \diamond_{\alpha} t \\
\leq & \left(\int_{a}^{b} w(t) f^{p}(t) \diamond_{\alpha} t\right)^{1 / p}\left(\int_{a}^{b} w(t) g^{q}(t) \diamond_{\alpha} t\right)^{1 / q}, \\
\int_{a}^{b} w(t) f(t) g(t) \diamond_{\alpha} t \leq & \left(\int_{x}^{b} w(t) f^{p}(t) \diamond_{\alpha} t\right)^{1 / p}\left(\int_{x}^{b} w(t) g^{q}(t) \diamond_{\alpha} t\right)^{1 / q} \\
& +\int_{a}^{x} w(t) f(t) g(t) \diamond_{\alpha} t \\
\leq & \left(\int_{a}^{b} w(t) f^{p}(t) \diamond_{\alpha} t\right)^{1 / p}\left(\int_{a}^{b} w(t) g^{q}(t) \diamond_{\alpha} t\right)^{1 / q}
\end{aligned}
$$

and

$$
\begin{aligned}
\int_{a}^{b} w(t) f(t) g(t) \diamond_{\alpha} t \leq & \frac{1}{2}\left(\left(\int_{a}^{x} w(t) f^{p}(t) \diamond_{\alpha} t\right)^{1 / p}\left(\int_{a}^{x} w(t) g^{q}(t) \diamond_{\alpha} t\right)^{1 / q}\right. \\
& +\int_{a}^{b} w(t) f(t) g(t) \diamond_{\alpha} t \\
& \left.+\left(\int_{a}^{x} w(t) f^{p}(t) \diamond_{\alpha} t\right)^{1 / p}\left(\int_{a}^{x} w(t) g^{q}(t) \diamond_{\alpha} t\right)^{1 / q}\right) \\
\leq & \left(\int_{a}^{b} w(t) f^{p}(t) \diamond_{\alpha} t\right)^{1 / p}\left(\int_{a}^{b} w(t) g^{q}(t) \diamond_{\alpha} s\right)^{1 / q}
\end{aligned}
$$

If $p<1$, then the inequalities (7), (8) and (9) are backwards.

Proof. (i)

(1) Suppose that $p>1$. Let $x_{1}, x_{2} \in \mathbb{T}, x_{1}<x_{2}<b$. Using the relation $\frac{1}{p}+\frac{1}{q}=1$, for $i=1,2$ we have 


$$
\begin{aligned}
\left(\int_{x_{i}}^{b} w(t) f^{p}(t) \diamond_{\alpha} t\right)^{1 / p}\left(\int_{x_{i}}^{b} w(t) g^{q}(t) \diamond_{\alpha} t\right)^{1 / q} & =\left(\int_{x_{i}}^{b} w(t) g^{q}(t) \diamond_{\alpha} t\right) \\
& \cdot\left(\frac{\int_{x_{i}}^{b} w(t) f^{p}(t) \diamond_{\alpha} t}{\int_{x_{i}}^{b} w(t) g^{q}(t) \diamond_{\alpha} t}\right)^{1 / p}
\end{aligned}
$$

The function $x \mapsto x^{1 / p}$ is concave and, using Jensen's inequality for concave functions, we have

$$
\begin{aligned}
\left(\int_{x_{1}}^{b} w(t) g^{q}(t) \diamond_{\alpha} t\right)\left(\frac{\int_{x_{1}}^{b} w(t) f^{p}(t) \diamond_{\alpha} t}{\int_{x_{1}}^{b} w(t) g^{q}(t) \diamond_{\alpha} t}\right)^{1 / p} & \left(\int_{x_{1}}^{b} w(t) g^{q}(t) \diamond_{\alpha} t\right)\left(\frac{\int_{x_{2}}^{b} w(t) g^{q}(t) \diamond_{\alpha} t}{\int_{x_{1}}^{b} w(t) g^{q}(t) \diamond_{\alpha} t} \cdot \frac{\int_{x_{2}}^{b} w(t) f^{p}(t) \diamond_{\alpha} t}{\int_{x_{2}}^{b} w(t) g^{q}(t) \diamond_{\alpha} t}\right. \\
& \left.\quad+\frac{\int_{x_{1}}^{x_{2}} w(t) g^{q}(t) \diamond_{\alpha} t}{\int_{x_{1}}^{b} w(t) g^{q}(t) \diamond_{\alpha} t} \cdot \frac{\int_{x_{1}}^{x_{2}} w(t) f^{p}(t) \diamond_{\alpha} t}{\int_{x_{1}}^{x_{2}} w(t) g^{q}(t) \diamond_{\alpha} t}\right)^{1 / p} \\
\geq & \left(\int_{x_{2}}^{b} w(t) g^{q}(t) \diamond_{\alpha} t\right)\left(\frac{\int_{x_{2}}^{b} w(t) f^{p}(t) \diamond_{\alpha} t}{\int_{x_{2}}^{b} w(t) g^{q}(t) \diamond_{\alpha} t}\right)^{1 / p} \\
& +\left(\int_{x_{1}}^{x_{2}} w(t) g^{q}(t) \diamond_{\alpha} t\right)\left(\frac{\int_{x_{1}}^{x_{2}} w(t) f^{p}(t) \diamond_{\alpha} t}{\int_{x_{1}}^{x_{2}} w(t) g^{q}(t) \diamond_{\alpha} t}\right)^{1 / p}
\end{aligned}
$$

Using, once again Jensen's inequality for concave functions on time scales, we get 


$$
\begin{aligned}
\left(\frac{\int_{x_{1}}^{x_{2}} w(t) f^{p}(t) \diamond_{\alpha} t}{\int_{x_{1}}^{x_{2}} w(t) g^{q}(t) \diamond_{\alpha} t}\right)^{1 / p}= & \left(\frac{1}{\int_{x_{1}}^{x_{2}} w(t) g^{q}(t) \diamond_{\alpha} t}\right. \\
& \left.\cdot \int_{x_{1}}^{x_{2}} w(t) g^{q}(t)\left(f(t)(g(t))^{-1 /(p-1)}\right)^{p} \diamond_{\alpha} t\right)^{1 / p} \\
\geq & \frac{1}{\int_{x_{1}}^{x_{2}} w(t) g^{q}(t) \diamond_{\alpha} t} \\
& \cdot \int_{x_{1}}^{x_{2}} w(t) g^{q}(t)\left(\left(f(t)(g(t))^{-1 /(p-1)}\right)^{p}\right)^{1 / p} \diamond_{\alpha} t \\
= & \frac{\int_{x_{1}}^{x_{2}} w(t) f(t) g(t) \diamond_{\alpha} t}{\int_{x_{1}}^{x_{2}} w(t) g^{q}(t) \diamond_{\alpha} t} .
\end{aligned}
$$

Using (10), (11) and (12), it follows

$$
\begin{aligned}
h\left(x_{1}, b\right)= & \left(\int_{x_{1}}^{b} w(t) f^{p}(t) \diamond_{\alpha} t\right)^{1 / p}\left(\int_{x_{1}}^{b} w(t) g^{q}(t) \diamond_{\alpha} t\right)^{1 / q} \\
& -\int_{x_{1}}^{b} w(t) f(t) g(t) \diamond_{\alpha} t \\
\geq & \left(\int_{x_{2}}^{b} w(t) g^{q}(t) \diamond_{\alpha} t\right)\left(\frac{\int_{x_{2}}^{b} w(t) f^{p}(t) \diamond_{\alpha} t}{\int_{x_{2}}^{b} w(t) g^{q}(t) \diamond_{\alpha} t}\right)^{1 / p} \\
& +\int_{x_{1}}^{x_{2}} w(t) f(t) g(t) \diamond_{\alpha} t-\int_{x_{1}}^{b} w(t) f(t) g(t) \diamond_{\alpha} t \\
= & \left(\int_{x_{2}}^{b} w(t) f^{p}(t) \diamond_{\alpha} t\right)^{1 / p}\left(\int_{x_{2}}^{b} w(t) g^{q}(t) \diamond_{\alpha} t\right)^{1 / q} \\
& -\int_{x_{2}}^{b} w(t) f(t) g(t) \diamond_{\alpha} t \\
= & h\left(x_{2}, b\right) .
\end{aligned}
$$

If $x_{2}=b$, the inequality (7) implies

$$
h\left(x_{2}, b\right)=h(b, b)=0 \leq h\left(x_{1}, b\right) .
$$

The result of the last inequality (14) is the decreasing monotony of the function $h(x, b)$ on $\mathbb{T}$, in relation with $x$. 
(2) Suppose that $p<1$. If $0<p<1$, then the function $x \mapsto x^{1 / p}$ is convex so that the inequalities from (7), (11) and (12) are reversed. This implies that (13) and (14) are also reversed, hence $h(x, b)$ is monotonously increasing over $\mathbb{T}$ in relation with $x$. If $p<0$, then $0<q<1$ and using the same arguments as for the case $0<p<1$, we obtain the increasing monotony of the function $h(x, b)$ over $\mathbb{T}$ in relation with $x$.

(ii) Using the same arguments as before, we can prove that the function $h(a, y)$ is monotonous in relation with $y$.

(iii) For every $x \in \mathbb{T}, a<x<b$ and $p>1$, the function $h(a, y)$ is monotonously increasing over $\mathbb{T}$ in relation with $y$ so that, it follows

$$
h(a, b) \geq h(a, x) \geq h(a, a)=0,
$$

meaning that

$$
\begin{aligned}
& \left(\int_{a}^{b} w(t) f^{p}(t) \diamond_{\alpha} t\right)^{1 / p}\left(\int_{a}^{b} w(t) g^{q}(s) \diamond_{\alpha} t\right)^{1 / q} \\
& \quad-\int_{a}^{b} w(t) f(t) g(t) \diamond_{\alpha} t \\
& \geq\left(\int_{a}^{x} w(t) f^{p}(t) \diamond_{\alpha} t\right)^{1 / p}\left(\int_{a}^{x} w(t) g^{q}(t) \diamond_{\alpha} t\right)^{1 / q} \\
& \quad-\int_{a}^{x} w(t) f(t) g(t) \diamond_{\alpha} t \geq 0
\end{aligned}
$$

and, adding $\int_{a}^{b} w(t) f(t) g(t) \diamond_{\alpha} t$ to each side of the previous inequality, we get (7).

Because $h(x, b)$ is monotonously decreasing over $\mathbb{T}$ in relation with $x$, we have

$$
h(a, b) \geq h(x, b) \geq h(b, b)=0,
$$


meaning

$$
\begin{aligned}
& \left(\int_{a}^{b} w(t) f^{p}(t) \diamond_{\alpha} t\right)^{1 / p}\left(\int_{a}^{b} w(t) g^{q}(t) \nabla_{\alpha} t\right)^{1 / q} \\
& \quad-\int_{a}^{b} w(t) f(t) g(t) \diamond_{\alpha} t \\
& \geq\left(\int_{x}^{b} w(t) f^{p}(t) \diamond_{\alpha} t\right)^{1 / p}\left(\int_{x}^{b} w(t) g^{q}(t) \diamond_{\alpha} t\right)^{1 / q} \\
& \quad-\int_{x}^{b} w(t) f(t) g(t) \nabla_{\alpha} t \geq 0
\end{aligned}
$$

and, adding $\int_{a}^{b} w(t) f(t) g(t) \diamond_{\alpha} t$ to each side of the previous inequality, we get (8). Adding the inequalities from (7) and (8), we obtain (9).

If $p<1$, the inequalities from (7), (8) and (9) are reversed, which implies that the inequalities from (15) and 16) are also reversed.

\section{Applications}

If $\mathbb{T}=\mathbb{R}$ and $w \equiv 1$ then Theorem 3 becomes Theorem 1.2 from [12]. If $\mathbb{T}=\mathbb{Z}$, $a=0, b=n, \alpha=0$ and $f(i)=a_{i}, g(i)=b_{i}, w(i)=b_{i}$ for $i=1, \ldots, n$, we have the following corollary that improves Theorem 1.1 from [12], if we note $H(n)=h(0, n)$ :

Corollary 4. Let $a_{i}>0, b_{i}>0, w_{i}>0(i=1,2, \ldots, n ; n>1)$ and consider $p, q$ as Hölder conjugates. If $p>1$, then it follows

$$
H(n) \geq H(n-i)
$$

If we define $C$ and $D$ by

$$
C(k)=\left(\sum_{i=1}^{k} w_{i} a_{i}^{p}\right)^{1 / p}\left(\sum_{i=1}^{k} w_{i} b_{i}^{q}\right)^{1 / q}+\sum_{i=k+1}^{n} w_{i} a_{i} b_{i}
$$

and

$$
D(k)=\left(\sum_{i=k+1}^{n} w_{i} a_{i}^{p}\right)^{1 / p}\left(\sum_{i=k+1}^{n} w_{i} b_{i}^{q}\right)^{1 / q}+\sum_{i=1}^{k} w_{i} a_{i} b_{i}
$$


for $k=1,2, \ldots, n$, then

$$
\begin{aligned}
& \sum_{i=1}^{n} w_{i} a_{i} b_{i}=C(1) \leq C(2) \leq \ldots \leq C(n-1)=\left(\sum_{i=1}^{k} w_{i} a_{i}^{p}\right)^{1 / p}\left(\sum_{i=1}^{k} w_{i} b_{i}^{q}\right)^{1 / q} \\
& \text { and } \\
& \sum_{i=1}^{n} w_{i} a_{i} b_{i}=D(n) \leq D(n-1) \leq \ldots \leq D(1)=\left(\sum_{i=1}^{k} w_{i} a_{i}^{p}\right)^{1 / p}\left(\sum_{i=1}^{k} w_{i} b_{i}^{q}\right)^{1 / q} .
\end{aligned}
$$

Also, for all $m=1, \ldots, n-1$, we have

$$
\sum_{i=1}^{n} w_{i} a_{i} b_{i} \leq \frac{1}{2}(C(m)+C(n-m)) \leq\left(\sum_{i=1}^{k} w_{i} a_{i}^{p}\right)^{1 / p}\left(\sum_{i=1}^{k} w_{i} b_{i}^{q}\right)^{1 / q}
$$

and

$$
\sum_{i=1}^{n} w_{i} a_{i} b_{i} \leq \frac{1}{2}(D(m)+D(n-m))\left(\sum_{i=1}^{k} w_{i} a_{i}^{p}\right)^{1 / p}\left(\sum_{i=1}^{k} w_{i} b_{i}^{q}\right)^{1 / q} .
$$

If $p<1$, then the above inequalities are reversed.

If $\mathbb{T}=\mathbb{Z}, a=0, b=n$ and $f(i)=a_{i}, g(i)=b_{i}, w(i)=w_{i}$, for $i=0, \ldots, n$, then, for all $\alpha \in[0,1]$, we get:

$$
\begin{aligned}
\int_{a}^{b} w(t) f(t) g(t) \diamond_{\alpha} t & =\alpha \sum_{i=0}^{n-1} w_{i} a_{i} b_{i}+(1-\alpha) \sum_{1}^{n} w_{i} a_{i} b_{i} \\
& =\alpha w_{0} a_{0} b_{0}+(1-\alpha) w_{n} a_{n} b_{n}+\sum_{1}^{n-1} w_{i} a_{i} b_{i}
\end{aligned}
$$

while,

$$
\begin{aligned}
\left(\int_{a}^{b} w(t) f^{p}(t) \diamond_{\alpha} t\right)^{1 / p} & =\left(\alpha \sum_{i=0}^{n-1} w_{i} a_{i}^{p}+(1-\alpha) \sum_{i=1}^{n} w_{i} a_{i}^{p}\right)^{1 / p} \\
& =\left(\alpha w_{0} a_{0}^{p}+(1-\alpha) w_{n} a_{n}^{p}+\sum_{i=1}^{n-1} a_{i}^{p}\right)^{1 / p}
\end{aligned}
$$


and

$$
\begin{aligned}
\left(\int_{a}^{b} w(t) g^{q}(t) \diamond_{\alpha} t\right)^{1 / q} & =\left(\alpha \sum_{i=0}^{n-1} w_{i} b_{i}{ }^{q}+(1-\alpha) \sum_{i=1}^{n} w_{i} b_{i}{ }^{q}\right)^{1 / q} \\
& =\left(\alpha w_{0} b_{0}{ }^{q}+(1-\alpha) w_{n} b_{n}{ }^{q}+\sum_{1}^{n-1} w_{i} b_{i}{ }^{q}\right)^{1 / q}
\end{aligned}
$$

Then, Theorem 3 proves, for every $0<k<n$ that

$$
\begin{aligned}
\alpha \sum_{i=0}^{n-1} w_{i} a_{i} b_{i}+(1-\alpha) \sum_{i=1}^{n} w_{i} a_{i} b_{i} & \left(\alpha \sum_{i=0}^{k-1} w_{i} a_{i}{ }^{p}+(1-\alpha) \sum_{i=1}^{k} w_{i} a_{i}{ }^{p}\right)^{1 / p} \\
\leq & \left(\alpha \sum_{i=0}^{k-1} w_{i} b_{i}{ }^{q}+(1-\alpha) \sum_{i=1}^{k} w_{i} b_{i}{ }^{q}\right)^{1 / q} \\
& +\alpha \sum_{i=k}^{n-1} w_{i} a_{i} b_{i}+(1-\alpha) \sum_{i=k+1}^{n} w_{i} a_{i} b_{i} \\
\leq & \left(\sum_{i=0}^{n-1} w_{i} a_{i}{ }^{p}+(1-\alpha) \sum_{i=1}^{n} w_{i} a_{i}{ }^{p}\right)^{1 / p} \\
& \cdot\left(\alpha \sum_{i=0}^{n-1} w_{i} b_{i}{ }^{q}+(1-\alpha) \sum_{i=1}^{n} w_{i} b_{i}^{q}\right)^{1 / q}
\end{aligned}
$$


hence,

$$
\begin{aligned}
\alpha w_{0} a_{0} b_{0}+(1-\alpha) w_{0} a_{n} b_{n}+\sum_{i=1}^{n-1} w_{i} a_{i} b_{i} & \left(\alpha w_{0} a_{0}{ }^{p}+(1-\alpha) w_{k} a_{k}{ }^{p}+\sum_{i=1}^{k-1} w_{i} a_{i}{ }^{p}\right)^{1 / p} \\
\leq & \left(\alpha w_{0} b_{0}{ }^{q}+(1-\alpha) w_{n} b_{n}{ }^{q}+\sum_{i=1}^{k-1} w_{i} b_{i}{ }^{q}\right)^{1 / q} \\
& +\alpha \sum_{i=k}^{n-1} w_{i} a_{i} b_{i}+(1-\alpha) \sum_{i=k+1}^{n} w_{i} a_{i} b_{i} \\
\leq & \left(\alpha w_{0} a_{0}{ }^{p}+(1-\alpha) w_{n} a_{n}{ }^{p}+\sum_{i=1}^{n-1} w_{i} a_{i}{ }^{p}\right)^{1 / p} \\
& \left(\alpha w_{0} b_{0}{ }^{q}+(1-\alpha) w_{n} b_{n}{ }^{q}+\sum_{i=1}^{n-1} b_{i}{ }^{q}\right)^{1 / q}
\end{aligned}
$$

and, also

$$
\begin{aligned}
\alpha w_{0} a_{0} b_{0}+(1-\alpha) w_{n} a_{n} b_{n}+\sum_{i=1}^{n-1} w_{i} a_{i} b_{i} \\
\leq\left(\alpha w_{k} a_{k}^{p}+(1-\alpha) w_{n} a_{n}{ }^{p}+\sum_{i=k+1}^{n} w_{i} a_{i}^{p}\right)^{1 / p} \\
\\
\quad\left(\alpha w_{k} b_{k}{ }^{q}+(1-\alpha) w_{n} b_{n}{ }^{q}+\sum_{i=k+1}^{n} w_{i} b_{i}{ }^{q}\right)^{1 / q} \\
+\alpha \sum_{i=0}^{k-1} w_{i} a_{i} b_{i}+(1-\alpha) \sum_{i=1}^{k} w_{i} a_{i} b_{i} \\
\leq\left(\alpha w_{0} a_{0}{ }^{p}+(1-\alpha) w_{n} a_{n}{ }^{p}+\sum_{i=1}^{n-1} w_{i} a_{i}{ }^{p}\right)^{1 / p} \\
\quad\left(\alpha w_{0} b_{0}{ }^{q}+(1-\alpha) w_{n} b_{n}{ }^{q}+\sum_{i=1}^{n-1} w_{i} b_{i}{ }^{q}\right)^{1 / q}
\end{aligned}
$$

The inequalities from (22) and (23) are improvements of the inequalities 
from (18), (19), (20) and (21).

(iv) If $\mathbb{T}=2^{\mathbb{N}}$ with $a=0, b=n$ and $f(i)=2^{a_{i}}, g(i)=2^{b_{i}}, w(i)=2^{w_{i}}$, where $a_{i}, b_{i}, w_{i} \in \mathbb{R}$, for $i=0, \ldots, n$, then for all $\alpha \in[0,1]$, we get:

$$
\begin{aligned}
\alpha 2^{w_{0}+a_{0}+b_{0}}+(1-\alpha) 2^{w_{n}+a_{n}+b_{n}}+\sum_{i=1}^{n-1} 2^{w_{i}+a_{i}+b_{i}} \\
\leq\left(\alpha 2^{w_{0}+p a_{0}}+(1-\alpha) 2^{w_{k}+p a_{k}}+\sum_{i=1}^{k-1} 2^{w_{i}+p a_{i}}\right)^{1 / p} \\
\cdot\left(\alpha 2^{w_{0}+q b_{0}}+(1-\alpha) 2^{w_{n}+q b_{n}}+\sum_{i=1}^{k-1} 2^{w_{i}+q b_{i}}\right)^{1 / q} \\
+\alpha \sum_{i=k}^{n-1} 2^{w_{i}+a_{i}+b_{i}}+(1-\alpha) \sum_{i=k+1}^{n} 2^{w_{i} a_{i} b_{i}} \\
\leq\left(\alpha 2^{w_{0}+p a_{0}}+(1-\alpha) 2^{w_{n}+p a_{n}}+\sum_{i=1}^{n-1} 2^{w_{i}+p a_{i}}\right)^{1 / p} \\
\quad \cdot\left(\alpha 2^{w_{0}+q b_{0}}+(1-\alpha) 2^{w_{n}+q b_{n}}+\sum_{i=1}^{n-1} 2^{w_{i}+q b_{i}}\right)^{1 / q}
\end{aligned}
$$


and, also

$$
\begin{aligned}
\alpha 2^{w_{0} a_{0} b_{0}}+(1-\alpha) 2^{w_{n} a_{n} b_{n}}+\sum_{i=1}^{n-1} 2^{w_{i} a_{i} b_{i}} & \left(\alpha 2^{w_{k}+p a_{k}}+(1-\alpha) 2^{w_{n}+p a_{n}}+\sum_{i=k+1}^{n} 2^{w_{i}+p a_{i}}\right)^{1 / p} \\
\leq & \left(\alpha 2^{w_{k} q b_{k}}+(1-\alpha) 2^{w_{n}+q b_{n}}+\sum_{i=k+1}^{n} 2^{w_{i}+q b_{i}}\right)^{1 / q} \\
& +\alpha \sum_{i=0}^{k-1} 2^{w_{i} a_{i} b_{i}}+(1-\alpha) \sum_{i=1}^{k} 2^{w_{i} a_{i} b_{i}} \\
\leq & \left(\alpha 2^{w_{0}+p a_{0}}+(1-\alpha) 2^{w_{n}+p a_{n}}+\sum_{i=1}^{n-1} 2^{w_{i}+p a_{i}}\right)^{1 / p} \\
& \cdot\left(\alpha 2^{w_{0}+q b_{0}}+(1-\alpha) 2^{w_{n}+q b_{n}}+\sum_{i=1}^{n-1} 2^{w_{i}+q b_{i}}\right)^{1 / q}
\end{aligned}
$$

\section{References}

[1] R. P. Agarwal, M. Bohner, Basic calculus on time scales and some of its applications, Results Math. 35 (1999), 3-22.

[2] M. Bohner and A. Peterson, Dynamic Equations on Time Scales, An introduction with Applications, Birkhäuser, Boston, 2001.

[3] C. Dinu, A weighted Hermite-Hadamard inequality for SteffensenPopoviciu and Hermite-Hadamard weights on time scales, Analele Ştiinţifice ale Universităţii "Ovidius”, Constanţa, Ser. Mat., 17 (2009).

[4] C. Dinu, "Hermite-Hadamard Inequality on Time Scales", Journal of Inequalities and Applications, vol. 2008, Article ID 287947, 24 pages, 2008.

[5] S. Hilger, "Analysis on measure chains-a unified approach to continuous and discrete calculus", Results Math. 35 (1990), pp. 18-56.

[6] S. Hussain and M. Anwar, "On certain inequalities improving the Hermite-Hadamard inequality", J. Inequal. Pure and Appl. Math. (JIPAM) 8 (2007), Issue 2, Article no. 60. 
[7] C. P. Niculescu and L.-E. Persson, Convex functions and their applications. A contemporary approach, CMS Books in Mathematics vol. 23, Springer-Verlag, New York, 2006.

[8] M. R. S. Ammi, Rui A. C. Ferreira, and Delfim F. M. Torres, "Diamond- $\alpha$ Jensen's Inequality on Time Scales and Applications", Journal of Inequalities and Applications, vol. 2008, Article ID 576876, 13 pages, 2008.

[9] T. Popoviciu, "Notes sur les fonctions convexes d'ordre superieur" (IX), Bull. Math. Soc. Roum. Sci., 43 (1941), 85-141.

[10] J. W. Rogers Jr. and Q. Sheng, "Notes on the diamond- $\alpha$ dynamic derivative on time scales", J. Math. Anal. Appl., 326 (2007), no. 1, 228-241.

[11] Q. Sheng, M. Fadag, J. Henderson and J. M. Davis, "An exploration of combined dynamic derivatives on time scales and their applications", Nonlinear Anal. Real World Appl., 7 (2006), no. 3, 395-413.

[12] Liang-Cheng Wang, "Two Mappings Related to Hölder's Inequality", Univ. Beograd. Publ. Elektrotehn. Fak., Ser. Mat. 15 (2004), 91-96.

Cristian DINU,

Department of Computer Science,

University of Craiova,

Str. A. I. Cuza, 13200585 Craiova, Romania.

Email: c.dinu@yahoo.com

Mihai STANCU,

Department of Computer Science,

University of Craiova,

Str. A. I. Cuza, 13200585 Craiova, Romania.

Email: mihaissss@yahoo.com

Daniela DĂNCIULESCU,

Department of Computer Science,

University of Craiova,

Str. A. I. Cuza, 13200585 Craiova, Romania.

Email: danadanciulescu@yahoo.com 